\title{
The role of MRI and MR arthrography in evaluation of wrist pain
}

\author{
Mohamed Basiouny Alghannam, ${ }^{1,}$, Mohamed Ramadan El-Kholy ${ }^{1}$, Ashraf Anas Zytoon ${ }^{1}$, \\ Ayman Mohamed Ebied ${ }^{2}$ \\ ${ }^{1}$ Department of Radiodiagnosis, Faulty of Medicine, Menoufia University, Egypt \\ ${ }^{2}$ Department of Orthopedics, Faulty of Medicine, Menoufia University, Egypt
}

Email address:

Mohamedalghannam1986@gmail.com (M. B. Alghannam)

\section{To cite this article:}

Mohamed Basiouny Alghannam, Mohamed Ramadan El-Kholy, Ashraf Anas Zytoon, Ayman Mohamed Ebied. The Role of MRI and MR Arthrography in Evaluation of Wrist Pain. International Journal of Medical Imaging. Vol. 2, No. 6, 2014, pp. 149-155.

doi: $10.11648 /$ j.jimi.20140206.16

\begin{abstract}
Objectives: The aim of this work is to show the value of MRI and MRA in evaluation of patients suffering from wrist pain. Background: Wrist pain is a common clinical complaint. The most common causes of wrist pain are traumatic \& non traumatic abnormalities involving avascular necrosis, ganglia, and TFC lesions. MRI serves as a problem solving technique to assess the cause of wrist pain. It also plays an important prognostic role. MRA is an excellent tool in assessing TFC and wrist ligaments injury. Methods: This study was conducted on 60 patients complaining of chronic wrist pain. The patients were referred to the radiology department from the outpatient clinics and the orthopedics department of Menoufia University Hospital. All patients were examined by x-ray and conventional MRI. 15 patients with suspected triangular fibrocartilage complex (TFC) and/or ligament injury underwent MRA as well. Data of soft tissue and osseous lesions were collected and analyzed. Results: There were thirty four males and twenty six females, their ages ranged between $17 \& 59$ years (mean age, 38 years). Joint effusion, kienbock disease and ganglion cysts were the most common findings in conventional MRI . TFC injury was the most common finding in MRA. Conclusion: MRI has a dramatic impact on the diagnosis and assessment of variety of wrist disorders. It is non invasive and ideal modality to demonstrate the complex anatomy and pathological conditions of the wrist. MRA is an excellent tool in assessing TFC and ligaments injury.
\end{abstract}

Keywords: MRI, MRA, Wrist Pain

\section{Introduction}

Wrist pain is an extremely common complaint, and there are many common causes of this problem. It is important to make an accurate diagnosis of the cause of the symptoms so that appropriate treatment can be directed at the cause ${ }^{(\mathbf{1})}$.

Magnetic resonance imaging (MRI) has been used to evaluate the wrist since the first clinical introduction of this technique into imaging practice ${ }^{(2)}$.This non-invasive method allows the diagnosis of additional soft tissue lesions ${ }^{(3)}$.

Unlike bone fractures, ligament injuries are often initially overlooked. These injuries can lead to progressive instability with secondary deterioration of the wrist joint ${ }^{(4)}$. These lesions most frequently involve the scapholunate and lunotriquetral ligaments, also known as the intrinsic ligaments and the triangular fibrocartilaginous complex (TFCC). Surgical techniques directed at specific injury patterns have been proposed, and precise preoperative diagnosis is necessary ${ }^{(5)}$.

Combination of the advantages of conventional arthrography with the direct visualization of structures on magnetic resonance (MR) imaging made magnetic resonance arthrography (MRA) the preferred modality for imaging patients with internal derangement of the wrist in some of the centers ${ }^{(6)}$.

\section{Materials and Methods}

This study was performed between August 2012 to April 2014 and was conducted on 60 patients complaining of chronic wrist pain. There were thirty four males and twenty six females, their ages ranged between $17 \& 59$ years (mean age, 38 years). The patients were referred to the radiology department from the outpatient clinics and the orthopedics 
department of Menoufia University Hospital.

All patients included in this study were subjected to Plain radiographic examination of the wrist, using conventional $\mathrm{X}$ ray unit. In the following position:

- Postero- Anterior view.

- Lateral view.

- Scaphoid view.

The MRI machine used was Toshiba Exclart Vantage 1.5$\mathrm{T}$ scanner. The examination protocol included coronal, sagittal, and axial planes. Coronal and axial fast spin-echo T1-weighted (TR/TE, 400/15), coronal axial and sagittal fast spin-echo T2-weighted (TR/TE, 2000/70), coronal gradient (TR/TE, 435/15) and coronal STIR (TR/TE, 20000/48) sequences with a field of view of $12 \mathrm{~cm}$ were used. Slice thickness was $2.5 \mathrm{~mm}$ with $2 \mathrm{~mm}$ interslice gap. All our patients were examined by conventional MRI. 15 patients with suspected triangular fibrocartilage (TFC) and / or ligament injury underwent MRA as well. The same surface coil was used and T1 fat suppression sequences (TR 550, TE 24, FOV 10, SL 2.5, gap 0.3, MATRIX 256 x 516) in coronal, axial and sagittal planes were done.

Table (1). Conventional MRI findings.

\begin{tabular}{lll}
\hline Lesion & Frequency & Percentage \\
\hline Kienbock disease & 10 & $16.6 \%$ \\
Ganglion cyst & 10 & $16.6 \%$ \\
Joint effusion & 14 & $23.3 \%$ \\
Scaphoid fracture & 7 & $11.6 \%$ \\
Carpal tunnel syndrome & 4 & $6.6 \%$ \\
tenosynovitis & 3 & $4 \%$ \\
Bone island & 2 & $3.3 \%$ \\
Lunotriquetral osteoarthritis & 2 & $3.3 \%$ \\
Associated subcutaneous edema & 2 & $3.3 \%$ \\
Bone tumor & 2 & $3.3 \%$ \\
TFC and ligamentous injury & 16 & $26.66 \%$ \\
Other Fractures & 3 & $4 \%$ \\
\hline
\end{tabular}

\section{Results}

Fourty patients suffered from right wrist pain while twenty had left side pain. TFC and ligament injury was the most common pathologic finding (16 patients) followed by joint effusion (14 patients), kienbock disease (10 patients), and ganglion cysts (10 patients). Neoplasm (2 patients) and arthritis (2 patients) were the least common (table 1).

Regarding TFC and ligamentous injury, TFC was the most common pathologic finding (10 cases in MRI and 12 cases in MRA) followed by scapholunate ligmant (SLL) injury (4 cases in MRI and 9 cases in MRA) and lunotriquetral ligament (LTL) injury ( 2 cases in MRI and 9 cases in MRA) ( table 2 ).

Table (2). MRI and MRA findings in TFC and ligamentous injury.

\begin{tabular}{lllll}
\hline Ligamentous injury & MRI & MRA & X2 & P Value \\
\hline TFC & 10 & $12(20 \%)$ & 0.6818 & 0.4089 \\
SLL & $(16.66 \%)$ & & 3.3937 & 0.0654 \\
LTL & $4(6.66 \%)$ & $9(15 \%)$ & 3.0335 & 0.008 \\
\hline
\end{tabular}

In the study population, the frequency and percentage of ulnar variance whether negative $(25 \%)$ or positive $(8.33 \%)$ was estimated due to its impact on the triangular fibrocartilage, and other ligamentous structures (table 3 ).

Table (3). The frequency and percentage of ulnar variance.

\begin{tabular}{lll}
\hline Ulnar variance & Frequency & Percentage \\
\hline -ve & 15 & $25 \%$ \\
+ve & 5 & $8.33 \%$ \\
Neutral & 40 & $66.66 \%$ \\
Total & 60 & $100 \%$ \\
\hline
\end{tabular}

Comparative study between the MRI and MRA findings regarding TFC and ligamentous injury was done ( table 4).

Table (4). Comparative study matching the MRI results, with that of MRA regarding TFC, SL \& LT tears

\begin{tabular}{|c|c|c|c|c|c|c|c|c|c|}
\hline & MRI & MRA & $\mathbf{X} 2$ & $P$ value & SEN of MRI & SPE of MRI & $(+) P P V$ & $(-) N P V$ & $\mathrm{ACC}$ \\
\hline TFC tear & 10 & 12 & 0.68 & 0.408 & 83.33 & 100.00 & 100.00 & 60 & 86.66 \\
\hline SL tear & 4 & 9 & 3.39 & 0.065 & 44.44 & 100.00 & 100.00 & 54.54 & 66.66 \\
\hline LT tear & 2 & 9 & 7.03 & 0.008 & 22.22 & 100.00 & 100.00 & 46.15 & 53.33 \\
\hline
\end{tabular}

\section{Discussion}

The anatomy of the wrist is complex and its structures are small, necessitating high-contrast and high-resolution imaging. In recent years, magnetic resonance imaging (MRI), and magnetic resonance arthrography (MRA) have greatly matured and proven efficacious for the diagnosis of internal derangements of the wrist ${ }^{(7)}$.

Arthrography of the wrist has been shown to be effective in diagnosis of TFCC tears. Magnetic resonance imaging (MRI) is a useful tool in the imaging of the wrist, because of its superior soft-tissue contrast and multiplanar capability ${ }^{(8)}$. Combination of the advantages of conventional arthrography with the direct visualization of structures on magnetic resonance (MR) imaging made magnetic resonance arthrography (MRA) the preferred modality for imaging patients with internal derangement of the wrist ${ }^{(9)}$.

Our study included sixty patients; males represented $56.66 \%$ of all patients while females represented $43.33 \%$, with their age ranged from 17 to 59 years. This is near to the study of Suzanne et al, ${ }^{(10)}$ which was performed on 34 patients to determine if MRI can differentiate between occult ganglion and synovitis in the chronic painful wrist, with males represent $67.6 \%$ of cases and females represent $32.3 \%$.

In our study, the patients were subdivided into traumatic and non traumatic categories that included avascular necrosis, ganglia, TFC lesions, ligament lesions, arthritis, fractures, neoplasm, tendinopathy and neuropathy. This is in agreement 
with the study of Richard et al, ${ }^{(1)}$ in which different imaging studies were used systematically to determine the cause of the wrist pain which was subdivided into pain of traumatic or non traumatic origin.

In our study, the chief complaint was painful wrist $(100 \%)$ plus other less common symptoms like limitation of movement (30\%), swellings (16.6\%), tingling and numbness $(6.6 \%)$. History of trauma was recorded in $(53.33 \%)$ of patients and $(46.66 \%)$ without history of trauma, while in the study of Richard et al, ${ }^{(11)}$ patients with wrist pain during activity represented (24\%), paresthesia (14\%), burning pain at rest (29\%) and swellings (57\%).History of trauma represented in $(10 \%)$ and $(90 \%)$ without history of trauma.

In our study patients with Kienböck disease, their ages ranged between 22 to 40 years old similarly Horch et al, ${ }^{(12)}$ study mentioned that patients with Kienböck disease their ages ranged between 20 to 35 years old.

Patients with AVN of lunate bone appeared normal in plain radiographs in early cases (4 patients in our study) and appeared with increased bone density in advanced cases (6 cases in our study). This is similar to study of Seymour et al, (13) who stated that sclerosis of lunate bone appeared in late cases of AVN.

There was total agreement with our study and Seymour et $a l$, ${ }^{(13)}$ study that reported that in MRI, AVN of the lunate (kienbock's disease) is manifested in its earliest stage by marrow edema. In advanced stage, the lunate bone had low signal in all pulse sequences. AVN of the scaphoid (preiser's disease) showed also similar features.

In the study done by Hoglund et al, ${ }^{(14)}$ ganglion cysts were commoner in females between the ages 30 to 40 years. This is similar to patients with ganglion cysts that were included in our study females recorded 6 patients while males recorded 4 patients and their ages ranged between 17 to 30 years old.

Suzanne et al, ${ }^{(10)}$ concluded that all radiographs of the patients with ganglia in their study were normal in appearance. There was no evidence of soft-tissue shadow, calcification, or bone or joint abnormality. These findings are similar to our study.

In our study ganglion cysts evaluation by MRI showed low SI in T1WI, high in T2WI and bright SI in STIR. While In the study done by Pretorius et al, ${ }^{(15)}$, ganglion cysts evaluation by MRI showed intermediate SI on T1 (mostly due to turbid fluid content) and bright SI on T2WI.

In our study, patients presented with fractures, their ages were between 25 to 55 years old.while James et al, ${ }^{(16)}$ study recorded that carpal fractures were very uncommon before age 12 and after 45 years James et al, ${ }^{(16)}$ added also in their study that the most common carpal bone fractured is the scaphoid, which accounts for about $75 \%$ of carpal fractures. Similar to our study in which scaphoid fractures accounted for $70 \%$ of carpal bone fractures.

Hauck et al, ${ }^{(17)}$ in their study mentioned that the ulnar styloid fractures occurred as isolated injuries or in association with distal radius and other wrist fractures. If the fracture occurred at the base of the styloid the attachment to the (TFCC) is disrupted this is in agreement with our study as one case with ulnar styloid process fracture associated also with TFC tear.

In our study four cases of carpal tunnel syndrome complained from pain, tingling and numbness. Sternbach, ${ }^{(18)}$ in his study in carpal tunnel syndrome stated that carpal tunnel syndrome was the most common form of nerve neuropathies. It was common in middle aged females .Symptoms could be sensory and/or motor. It included numbness and tingling localized to the sensory distribution of the median nerve.

Leifer et al, ${ }^{(19)}$ reported in their study that causes of carpal tunnel syndrome (CTS) may be idiopathic due to congenital narrowing of the carpal tunnel. This is in near to our study.

In our study, MRI revealed median nerve had increased signal intensity in T2WI. Similarly to Pasternack et al, ${ }^{(20)}$ studies that in MRI median nerve had high signal intensity in $\mathrm{T} 2$ weighted images.

In our study the case with De-Quervain's syndrome, abductor polices longus (APL) and extensor polices brevis (EPB) tendons had tenosynovitis. This is in agreement with Bianch et al, ${ }^{(21)}$ study. They recorded that De-Quervain's syndrome was a stenosing tenosynovitis of the first dorsal compartment of the wrist which contains APL and EPB tendons.

In our study, MRI examination of painful wrist showed that tenosynovitis was manifested as fluid signal within the tendon sheath low signal intensity in T1 and high in T2WI, these features are shown best by axial images over the extent of the tendons. Seymour et al, ${ }^{(13)}$ and Glajchen \& Schweitzer, ${ }^{(22)}$ studies mentioned the same finding in MRI the affected tendon sheaths showed low signal intensity around the tendons in T1WI and intense bright in T2WI that evaluated best in axial pulse sequences.

In our study patients were examined first by nonenhancing conventional MRI, followed by MRA in 15 patients, for better delineation of the internal structures, and accurate diagnosis.

This agrees with Zeev et al, ${ }^{(9)}$ who stated that joint distension by contrast injection, visualization of contrast leakage, allows better evaluation of subtle abnormalities such as a partial ligamentous tear or cartilage defects.

Thomas et al, ${ }^{(23)}$ compared conventional MR imaging with MR arthrography and concluded that the accuracy of diagnosis can be improved with the addition of an intraarticular injection.

In our study injection was done through a dorsal approach, starting by the mid carpal compartment, where 7 patients displayed active leakage into the radio carpal joint, obviating the need for additional injection, while the remaining 8 patients showed no evidence of communication and additional injection into the radio carpal joint was done under fluoroscopic guidance.

Twelve patients showed leakage into the distal radioulnar joint (DRUJ) after radio carpal injection, denoting indirectly an existing defect within the TFC. Injection into the DRUJ was not done in our study because of the discomfort for the 
patients, and the time consuming factor.

Schmitt et al, ${ }^{(24)}$ agreed with double injection into the mid carpal and radio carpal joint compartments, while Suraj et al, (8) \& Lynne et al, ${ }^{(25)}$ agreed that single radio carpal injection followed by fat suppressed gadolinium sensitive sequences is sufficient.

Amrami, (26) considered the single compartment arthrography to be more definitive and that the interpretation of multi-compartment injections on the static MRI images obtained after arthrography is complex, since there is no available "subtraction" technique and it can be difficult to sort out which ligaments are completely or partially torn and what the direction of contrast flow has been.

Amrami, (26) preferred a single compartment injection planned with the referring surgeon, performing a single injection in the most clinically relevant compartment and then adding additional injections if a tear is not seen on the conventional arthrogram preceding MRI examination.

Marco et al, ${ }^{(27)}$, \& Theumann et al, ${ }^{(28)}$ both advocated the need for triple compartment injection for better delineation of peripheral partial TFC tears.

Our study was in accordance with Khoury et al, ${ }^{(7)}$ who found that the diagnostic performance of MRI is improved by MRA making lesions more conspicuous when they are outlined by contrast material in a distended joint space.

Also our study coincided with Thomas et al, ${ }^{(23)}$ who conducted their study on 45 patients, comparing the results of un-enahnced MRI, with both MRA \& MD CT arthrography, in their study un-enhanced MRI diagnosed eight central and three ulnar TFC tears, showing a sensitivity \& specificiy of $31-60 \%, \&$ 96-100\%. However our sensitivity $(83.33 \%)$ was higher than theirs.

As regard SLL, our study shows sensitivity of $44.44 \%$ and specificty of $100 \%$, while Thomas et al, 2007, shows specificity in the range of $72-94 \%$ and sensitivity in the range of $65-90 \%$.

Khoury et al, ${ }^{(7)}$ stated that generally, the sensitivity for the detection of SLL tears was found to be superior to that of LTL tears, which agrees with our results.

MR arthrography cannot replace arthroscopy; however, it could be a potent additional tool for wrist imaging. It can facilitate the diagnosis and the indication for surgery of the wrist and help to reduce arthroscopic interventions for purely diagnostic purposes and without any therapeutic consequences $^{(7)}$.

We believe that direct MR arthrographic imaging is well suited for detecting intra-articular lesions of the wrist. The presented diagnostic results of MR arthrography are superior to the results of un-enhanced MRI. Direct MR arthrography as a reliable diagnostic tool is strongly recommended if lesions of the scapholunate ligament and the triangular fibrocartilage complex are suspected ${ }^{(9)}$.

Chart (1) shows the results of conventional MRI and MRA in TFC and ligamentous injury, for example, 10 cases (16.6\%) of TFC tear were detected by MRI, while on MRA 12 cases $(20 \%)$ were detected.

Chart (2) shows the frequency of leakage from the mid carpal (MC) to the radio carpal (RC) joint (which denotes SLL and / or LTL injury) and the frequency of leakage from the radio carpal (RC) to the distal radio ulnar joint (DRUJ) which denotes TFC injury during intra-articular injection in the study population. $46.6 \%$ of cases show leakage from MC to $\mathrm{RC}$ joint and $80 \%$ of cases show leakage from $\mathrm{RC}$ to DRUJ.

Chart (3) shows the frequency and percentage of TFC and ligamentous injury in MRA cases. For example, 9 cases show SLL tear in MRA that represents about $60 \%$ of MRA cases.

Some of the cases are shown in figures. Figure 1 shows a case of kienbock disease (avascular necrosis of the lunate bone) with sclerosis of the lunate bone in plain $\mathrm{x}$-ray. The lunate shows hypointense signal in T1WI and heterogeneously hyperintense signal in T2WI and STIR images with reduced height of its radial aspect.

Figure 2 shows tendon related ganglion cyst that appears isointense in T1WI and hyperintense in T2WI, STIR and gradient images.

Figure 3 shows case of scaphoid fracture with bone ischemia. The fracture line is well seen in the plain x-ray and appears hypointense in T1WI. High signal is seen in the scaphoid bone in STIR image denoting marrow contusion and mild bone ischemia.

Figure 4 shows case of TFC tear that appears as area of altered signal intensity in coronal T2WI and gradient images. On coronal fat suppressed T1WI and T1 MR arthrographic images a defect is seen within the TFC with leakage of contrast into the DRUJ.

Figure 5 shows case of torn TFC and torn SLL and LTL. Coronal gradient image shows TFC tear and SLL tear. Coronal fat suppressed T1WI and T1 MR arthrographic show the TFC tear and torn SLL and LTL.

Figure 6 is coronal T1 MR Arthrographic image that revealed tear within the luno triquetral ligament.

\section{Conclusion}

The wrist joint is considered the most complex articulation in the human body. The small size of the anatomic structures, the diversity of disorders that can cause symptoms, and the high rate of asymptomatic findings are some of the factors that contribute to the difficulty of diagnosis and management in this region.

MR imaging has been used in the evaluation of a wide spectrum of this joint disorder, its multiplanar capabilities and refined tissue contrast allow detailed assessment of osseous and soft tissue pathology.

MRI presented significant advantage over other imaging modalities in assessing the tendon abnormalities. Other advantages included ability to image both bone and soft tissue satisfactorily, and the capability of direct multiplanar imaging.

The small ligaments of the wrist including the scapholunate interosseous ligament can be challenging to assess at conventional wrist MRI, especially given their complex 
anatomy.

Magnetic resonance arthrography (MRA) has become the preferred modality for imaging patients with internal derangement of the wrist, which include lesions of the triangular fibrocartilage complex (TFCC), scapholunate and lunotriquetral ligaments.

MRA (MR arthrography) combines the advantages of conventional MR imaging and arthrography by improving the visualization of small intra-articular abnormalities.

MR arthrography is an excellent technique for determining the site and extent of pathologic lesions of the TFCC, and the intrinsic ligaments. It is useful in the evaluation of patients who have refractory pain, or instability syndromes of the wrist.

We believe that we can now use MRA of the wrist in our department with excellent results without the need to perform unnecessary diagnostic arthroscopy.

In conclusion, arthroscopy continues to be considered the gold standard in the diagnosis of intrinsic ligaments and TFCC lesions. However, further refinements in MR technology will bring MRA closer to becoming the examination of choice for wrist internal derangement and might replace diagnostic arthroscopy.

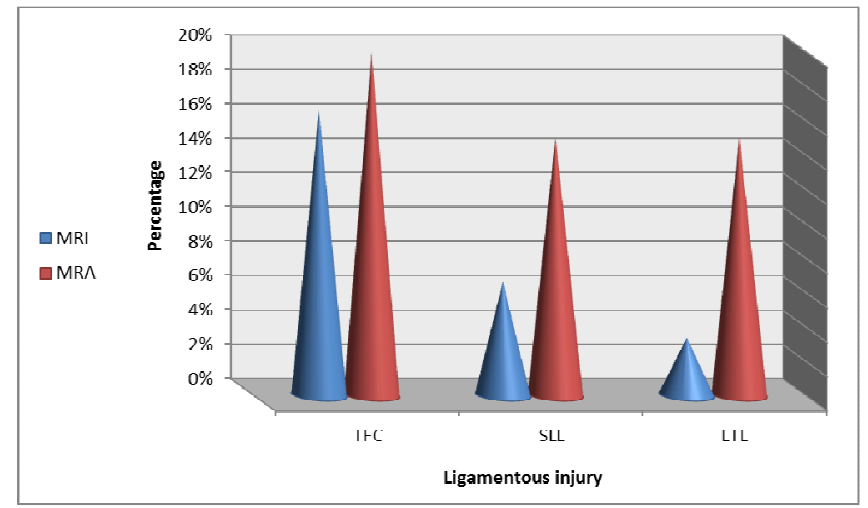

Chart (1). An illustration of conventional MRI and MRA findings in ligamentous injury

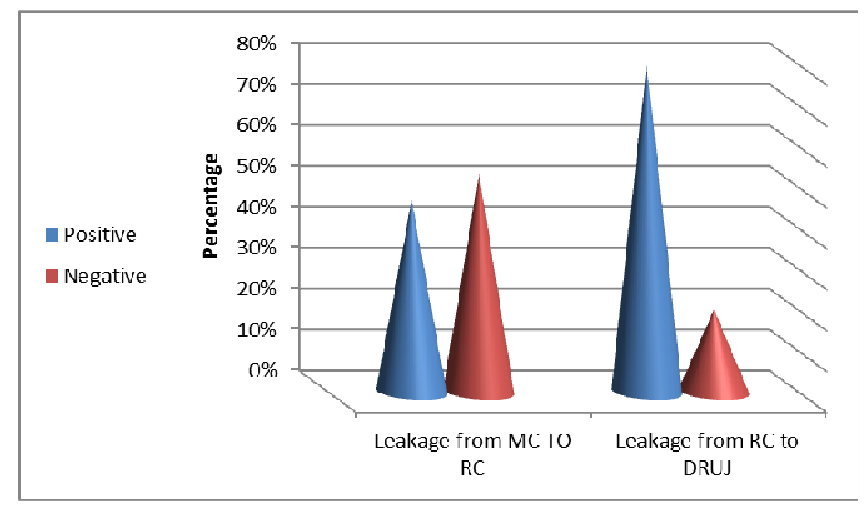

Chart (2). shows the frequency of leakage from the mid carpal $(M C)$ to the radio carpal $(R C)$ joint (which denotes SLL and / or LTL injury) and the frequency of leakage from the radio carpal $(R C)$ to the distal radio ulnar joint (DRUJ) which denotes TFC injury during intra-articular injection in the study population.

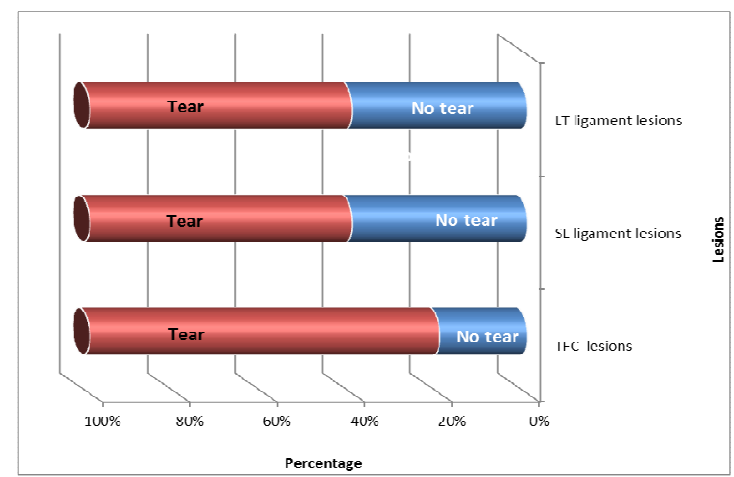

Chart (3). Shows the frequency and percentage of TFC and ligamentous injury in $M R A$

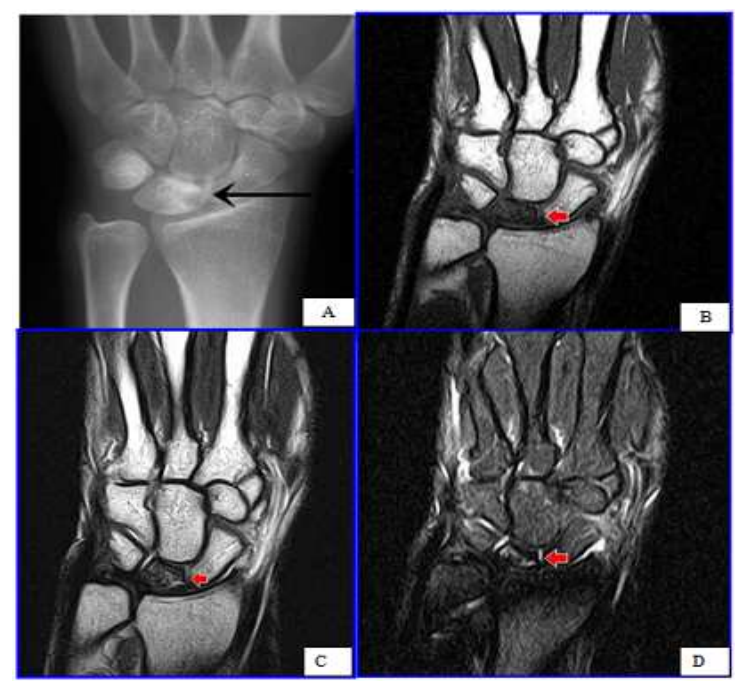

Figure No. (1). Plain X-ray, MRI coronal T1WI, coronal T2WI and STIR show Evidence of avascular necrosis of the lunate bone (A) Plain x-ray revealed sclerosis of the lunate bone with decreased height on the radial aspect of the bone. (B) Coronal T1WI shows hypointense signal within the lunate bone with decreased height on the radial aspect of the bone. $(C \& D)$ Coronal T2WI revealed heterogenous signal of the lunate bone with decreased height on the radial aspect of the bone .

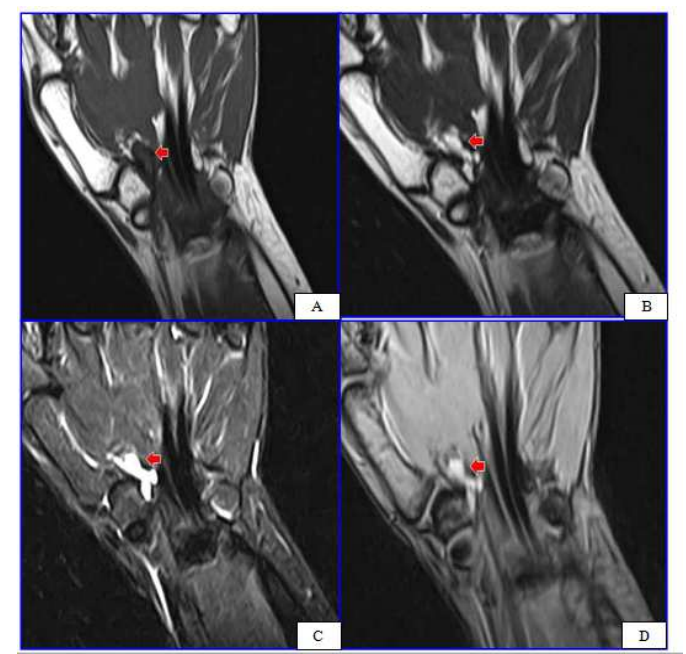

Figure No. (2). Coronal T1, T2, STIR and gradient WI showing ganglion cyst related to the flexor polices tendon (A) Coronal T1WI shows irregular shaped isointense structure related to the flexor pollicis tendon. $(B, C \& D)$ Coronal T2WI, STIR and gradient images show hyperintense signal within the lesion. 


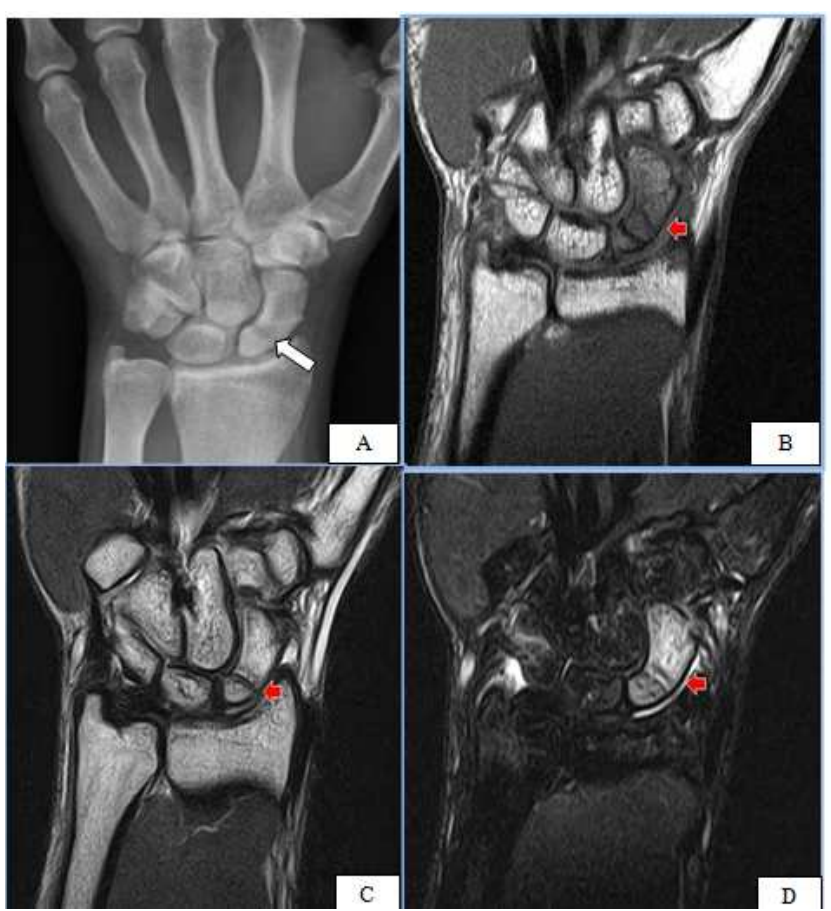

Figure No. (3). Plain X-ray, MRI coronal T1WI, coronal T2WI and STIR images show fracture scaphoid with bone ischemia. (A) Plain x-ray shows the fracture line within the scaphoid bone $(B)$ Coronal T1WI shows the fracture line within the scaphoid bone with hypointense signal of the scaphoid bone. (C) Coronal T2WI shows hypointense fracture line within the proximal part of the scaphoid bone with mildly increased signal of the rest of the scaphoid. (D) Coronal STIR image shows the fracture line and increased signal within the scaphoid bone denoting ischemia, minimal joint effusion could be detected

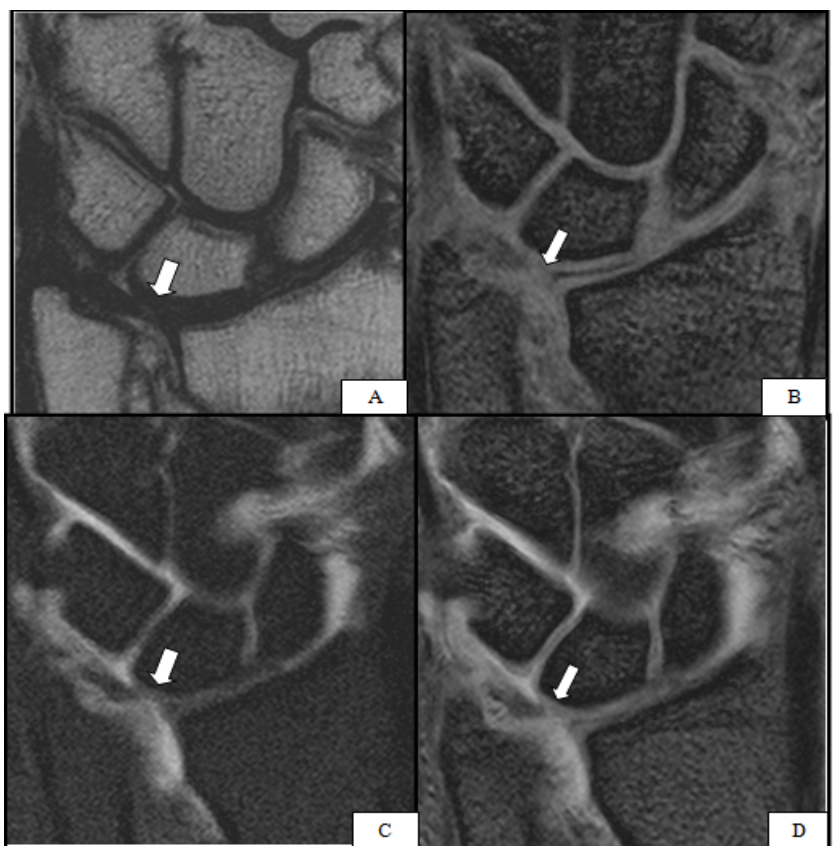

Figure No. (4). Coronal T2, gradient, and coronal fat suppressed T1 WI post contrast showing TFC tear. (A, B) Coronal $T 2 \&$ \& gradient images revealed an area of attenuation with altered signal within the TFC (arrow).(C\&D) Coronal Fat suppressed T1 WI (A) \& T1 MR Arthrographic images revealed a defect within the TFC (arrows), with leakage of the injected contrast into the DRUJ, delineating the tear.

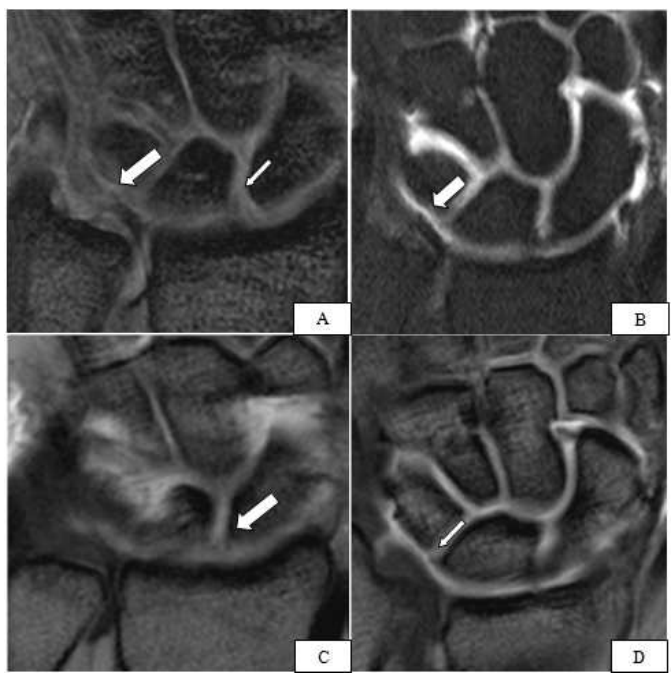

Figure No. (5). Coronal gradient, coronal fat suppressed T1 WI post contrast and coronal T1WI post contrast showing torn TFC, torn SLL and torn LTL. (A) Coronal T2 gradient image revealed intra substance central tear of the TFC, reaching the superior articular surface (large arrow), also tear of the SL ligament is noted (small arrow). (B) Coronal T1 Fat suppression MR Arthrogaphic image shows the central TFC tear less conspicuous than at the pre contrast images, yet some leakage of contrast is observed at the DRUJ. Coronal T1 MR Arthrographic images (C\&D) shows tears of the SL (thick arrow) \& LT (thin arrow) ligaments.

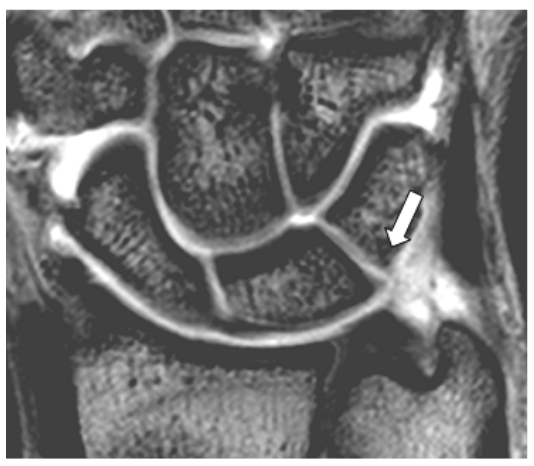

Figure No. (6). Coronal T1 MR Arthrographic image revealed tear within the luno triquetral ligament (arrow).

\section{References}

[1] Jonathan Cluett, M.D (2010) , About.com Guide Updated April 28, 2010

[2] Andrew H. Haims ,Andrew E. Moore ,Mark E. Schweitzer ,William B. Morrison ,Diane Deely ,Randall W. Culp and Howard P. Forman( 2004) ,AJR 2004;182:12671270

[3] Urbschat K, Frank J, Marzi I, Schneider G and Roth R (1999). Value of MRI in di-agnosis of post-traumatic wrist complaints. Zentralbl Chir 124:977-983

[4] Watson HK, Weinzweig J and Zeppieri J (1997). The natural progression of scaphoid instability. Hand Clin 1997 13:39-49

[5] Garcia-Elias M, Geissler W,Hotchkiss R, Pederson W and Wolfe S (2005) .Green's operative hand surgery. Philadelphia, PA Elsevier, 2005:535-604 
[6] Elentuck D and Palmer WE (2004). Direct magnetic resonance arthrography. Eur Radiol. 2004; 14:1956-67.

[7] Viviane Khoury, M.D., Patrick G. Harris, M.D. and E' tienne Cardinal, M.D. (2007). Cross-Sectional Imaging of Internal Derangement of the Wrist with Arthroscopic Correlation. Semin Musculoskelet Radiol; 11:36-47, 2007.

[8] Suraj Joshy, Kenneth Lee, Subodh C and Deshmukh (2008) . Accuracy of direct magnetic resonance arthrography. in the diagnosis of triangular fibrocartilage complex tears of the wrist International Orthopaedics (SICOT); 32:251-253, 2008.

[9] Zeev V. Maizlin, Jacqueline A. Brown, Jason J and Clement (2009). MR Arthrography of the Wrist: Controversies and Concepts. American Association for Hand Surgery; 4:66-73, 2009.

[10] Suzanne E., Anderson, Lynne S., et al. (2006). MRI for Differentiating Ganglion and Synovitis in the Chronic Painful Wrist AJR; 186:812-818, 2006.

[11] Richard M, van Vugt, Johannes W J, et al. (1999). Chronic wrist pain: diagnosis and management. Development and use of a new algorithm. Ann Rheum Dis; 58; 665-674, 1999.

[12] Horch RE, Unglaub F, Dragu A, et al.(2008). Kienbock's disease: Diagnosis and therapy. Chirurg Feb 13; epub ahead of print, 2008.

[13] Seymour. MRCP, Frcr PG, White, et al. (1998). Magnetic resonance imaging of painful wrist, the British journal of radiology; 7:1323-1330, 1998.

[14] Hoglund M, Tordai P and Muren C. (1994). Diagnosis of ganglions in the hand and wrist by sonography. Acta Radiol; 8:9-35, 1994.

[15] Pretorius ES, Epstein RE, Dalinka, et al.(1997). MK.MR imaging of the wrist .Radiol clin N Am; 35 :61-145.1997.

[16] James R.D., Murray Erskine J., Hoimes Rakesh, et al.(2008) . A-Z of Musculoskeletal and Trauma, Radiology Cambirdge University Press; 2:264-274, 2008.

[17] Hauck RM, Skahen J and Palmer AK (1996). Classification and treatment of ulnar styloid nonunion. J Hand Surg [Am]. May; 21(3):418-22, 1996.
[18] Sternbach G. (1999). The carpal tunnel syndrome. J Emerg Med; 17:23-519, 1999.

[19] Leifer D, Cros D, Halperin JJ, et al. (1992). Familial bilateral carpal tunnel syndrome: report of two families. Arch Med. Rehab.; 73:393-397, 1992.

[20] Pasternack I, Malmivara A, Kenn W, et al. (2003). MRI findings in respect to carpal tunnel syndrome .Euro. Radiol.; 10(7):1043-1050,2003.

[21] Bianch S, Martinoil C and Abdel Wahab IF.(1999). High Frequency Ultrasound examination of the wrist and hand .Skeletal Radiology, 28:121-129, 1999.

[22] Glajchen N and Schweitzer M. (2004). MRI features of De Quervain tenosynovitis of the wrist. Skeletal Radiology; 6365, 2004.

[23] Thomas Moser, Jean-Claude Dosch and Akli Moussaoui (2007). Wrist Ligament Tears: Evaluation of MRI and Combined MDCT and MR Arthrography. AJR; 188:12781286, 2007.

[24] Schmitt R, Christopoulos G, Meier R, et al. (2003). Direct MRarthrography of the wrist in comparison with arthroscopy: a prospective study on 125 patients. Rofo; 175(7):911-9, 2003.

[25] Lynne S. Steinbach, MD, William E., et al. (2002). Special focus session, MR Arthrography. Radiographics, 22; 12231246, 2002.

[26] Amrami KK (2006). Magnetic resonance arthrography of the wrist: case presentation and discussion. J Hand Surg Am.; 31:669-72, 2006.

[27] Marco, Zanetti, Nadja, Saupe, Ladislav and Nagy (2007). Role of MR imaging in chronic wrist pain. Eur Radiol; 17: 927-938, 2007.

[28] Nicolas H. Theumann.MD, Ghazal Etechami.MD and Bertrand Duvoisin MD (2006). Association between Extrinsic and Intrinsic Carpal Ligament Injuries at MR Arthrography and Carpal Instability at Radiography: Initial Observations. Radiology; 238 (3): 950-957, 2006. 